\title{
Adaptive QoS for Mobile Multimedia Services over Wireless Networks
}

\author{
Alejandra Mercado and K.J. Ray Liu \\ Electrical and Computer Engineering Department \\ University of Maryland \\ College Park, MD 20742 \\ Tel:301-405-7319, email:santiago, kjrliu@eng. umd. edu
}

\begin{abstract}
A wireless network that provides multimedia services should control the quality of the transmission link for users, allocating resources according to the service type of each user, be it voice, video, image or data. This paper proposes a scheme to allocate the desired signal to interference noise ratio (SINR) for those users with multimedia services by exploiting power control and smart antennas, while not affecting users with voice service. A method to quickly initialize new users into such a network is also proposed.
\end{abstract}

\section{INTRODUCTION}

Wireless network technology has advanced prodigiously to the point where we can now talk of providing multimedia services to mobile telephony users. A wireless network with integrated multimedia services, requires that each user should have a different channel quality to accommodate different service types, say voice, video conferencing, email, etc. The SINR levels, however are strongly related to automatic power control and smart antenna results. Using these tools, we propose to control the levels of SINR that each user is afforded, and this selection is done according to the individual need of each user, and the quality of channels that the mobile system can allow, given the interference present at any given time.

We address two problems associated to this subject: the first is how to accommodate the service requirements for each user, given that each multimedia service type has a target SINR level, and the second is how to hasten the system processor through the necessary calculations to quickly provide the multimedia service to each user. The latter point arises when the system is initiating service for a new user, whether the new user is initiating a call or if it is handing off from another base station. During this process, the system must calculate the power vector for all cochannel mobile users, and the antenna weights for all the associated base stations. These values can be obtained through iterative algorithms that require certain constraints for convergence, but these constraints may be lengthy to verify. In a practical situation, an extensive delay before activating the new users is not allowable. We present a technique which reduces the activation latency time for new multimedia service users. For this, we propose a fast and coarse method for finding the mobile powers and antenna weights. The technique we present to address the former problem of increasing the SINR as much as possible, uses an iterative incremental procedure that takes the SINR for all multimedia users closer to their target SINR levels. This is done off-line, so there is no additional delay for any user.

The subject of joint automatic power control and smart antennas has been addressed in [1], where an RLS algorithm is used to track a CDMA channel in the presence of cochannel interference, and an analysis is made of the gain provided by the smart antenna configuration. This work, does not address the individual channel qualities, nor a proposal to control them. In [2], the TDMA mobile radio standard IS-136 is the platform used to study the coverage area increase provided by joint power control and smart antennas. There, however, the transmit power must remain constant during each time slot due to restrictions imposed by IS-136. Also, the individual SINR levels granted to each user is not addressed. Where the quality level for individual users was addressed was in [3], but here the perspective is of providing several parallel lower rate data streams. Here, for high data rate users, the data sequences are first converted to several parallel basic rate data streams, thus more resources are alloted to these users. Resource assignment for mobile cellular has been seen in [4] from the perspective of channel assignment, or more extensively in [5] with a broader set of resources. But in both cases, they attempt to provide users with the QoS that is available, without changing mobile powers or antenna gains to create a better QoS for the users who need it.

The other new issue that this paper addresses is that the existing trial and error techniques are computationally long [6], [2], and they may extend the time that new users must wait while powers and antenna weights are calculated. Our method exploits mathematical tools that allow us to quickly find a solution for the new values to be calculated. This, in turn, translates to a faster service for new users.

The system model is described in section II. The proposed techniques for quick activation and SINR fine-tuning are explained in section III. The complete algorithm is put together for the reader in section IV. Finally sections V and VI have simulation results and conclusions, respectively.

\section{General Model AND Algorithm}

Assume a wireless network with $M$ cochannel bases, which employs automatic power control and smart antennas. A diagram of the antenna weights, $w_{i}(j)$, can be appreciated in Fig. 1 , where the subindex $i$, indicates the base station index, and the index $j$ indicates each antenna element index. The output of each antenna element is the multiplications of the delayed signal by the antenna array response vector. The final output, $\mathbf{w}_{i}^{H} \mathbf{x}_{i}$, is the final received signal. The weights are chosen so as to minimize the overall antenna gain pattern towards all interfering cochannel sources, while keeping an acceptable gain towards the desired mobile, see Fig. 2. The automatic power control scheme attempts to minimize the overall power of all mobiles, while guaranteeing a certain SINR level for each user. If the SINR levels that are chosen are too high, the optimal power vector and antenna weights cannot be obtained.

Our goal is to assign the SINR levels, $\gamma_{i}$, according to each user's needs and test whether these SINR assignments allow us to achieve optimum power and antenna weight allocations for those $\gamma_{i}$.

The optimal solution for joint power control and smart antennas, for a fixed set of $\gamma_{i}$, has been solved in [6], where the performance measure that has been used for controlling the smart antennas is the minimum noise variance criterion [7]. If we define $\bar{\gamma}_{i}$ as the effective SINR for user $i$, then the desired constraint is $\tilde{\gamma}_{i} \geq \hat{\gamma}_{i}$ for all $i=1, \ldots M$, where $\hat{\gamma}_{i}$ is the target SINR for user $i$. In vector form, the constraint can be written as

$$
\mathbf{P}-\Gamma F \mathbf{P} \geq \mathbf{u},
$$

where $F$ is the pathgain ratio matrix,[8], $\mathbf{P}$ is the power vector for all mobiles, and

$$
\mathbf{u}=\left(\hat{\gamma}_{1} \frac{n_{1}}{G_{11}}, \cdots \hat{\gamma}_{M} \frac{n_{M}}{G_{M M}}\right)^{T}
$$




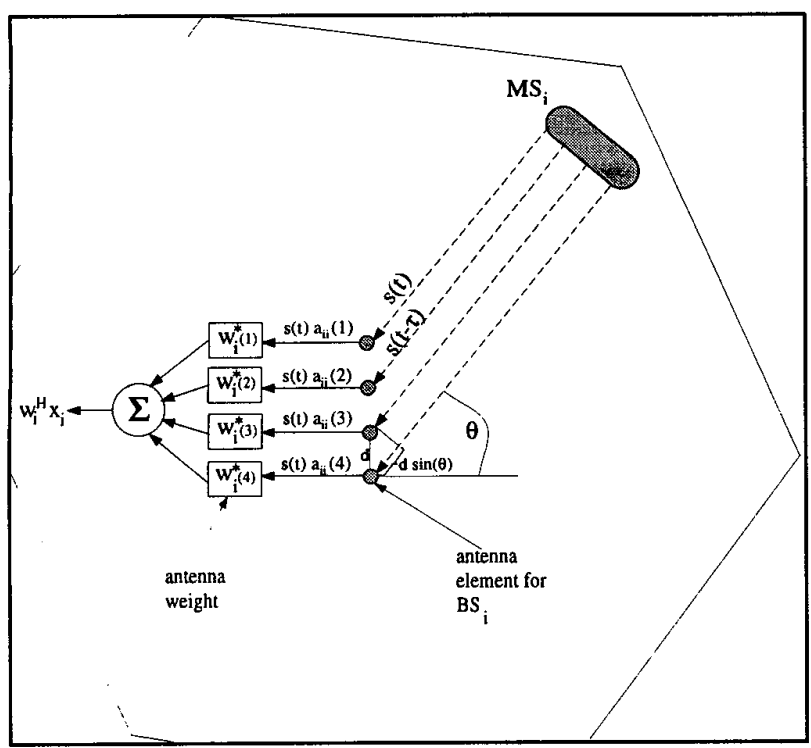

Fig. 1. Smart antenna input diagram. $M S_{i}$ represents mobile station $i$, and the base station has four antenna elements.

$n_{i}$ is white noise at base station $i$, and $G_{i j}$ is the pathgain between mobile $j$ and base station $i$. $\Gamma$ is a diagonal matrix with all $\hat{\gamma}_{i}$. The inequality is taken to be element by element.

The requirement for the attainment of the optimal vector $\mathbf{P}$ and antenna gains for the above situation is verifying the existence of any feasible set of weight vectors, $\left\{\mathbf{w}_{i}\right\}_{i=1, \ldots M}$ in the complex plane such that $\rho(\Gamma F)<1$, [6], [9], [10]. This problem, in itself is difficult; and it is further complicated for us since we must find them for the most favorable $\Gamma$ possible. Finding the optimum $\Gamma$ has been shown to be an NP-hard problem[11]. We present a suboptimal solution that reduces the problem to one of lower complexity.

\section{Methods for QUick ACTIVATION AND Fine TUNing}

This section describes how to quickly guarantee convergence for a joint power control/smart antenna calculation by fast determination of feasible SINR levels. This, in turn, allows the system to activate the mobile units quickly. After that, the system devotes its resources to fine-tuning the SINR levels for multimedia service users; the technique for this is also presented in this section.

\section{A. Fast Path to Convergence}

We've already seen that the test for convergence for the joint power control/beamforming solution requires scanning over all the complex plane for $M$ vectors that comply with $\mathbf{w}_{i}^{H} \mathbf{a}_{i i}=1$ and also have $\rho(\Gamma F)<1$. Here, $a_{i i}$ is the array response vector from base station $i$ to mobile $i$, as can be seen in Fig. 1. We wish to perform this test for the best possible $\Gamma$.

We propose to decouple the matrix $F$ and replace the spectral radius with the matrix norm one, which is faster to compute, and guarantees convergence when the spectral radius does, [10]. We must be cautioned that even though it is clear we can replace the spectral norm with this simpler norm one, the spectral radius should be used in the fine-tuning stage. This is because, though difficult to handle, the spectral radius is a more precise indicator of the convergence of the power control algorithms, since $\rho(A) \leq\|A\|$ for all consistent norms, [12].

Denote the collection of weights, pathgains, and antenna gains by $\mathcal{W}, \mathcal{G}, \mathcal{A}$, respectively. First, we decompose $\Gamma F$ into three matrices,

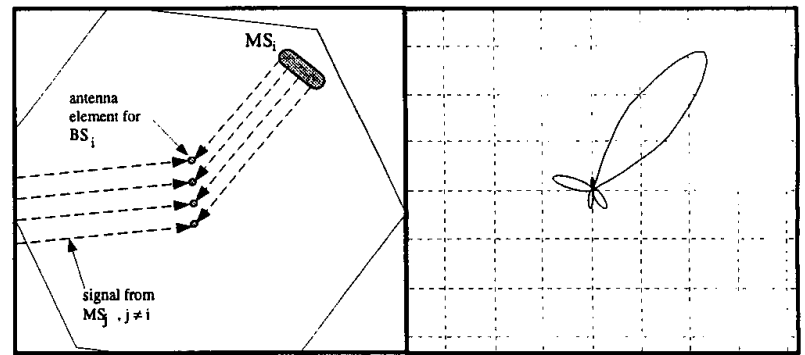

Fig. 2. The antenna beam former at the base station directs a large gain towards the desired signal and a small gain towards the interferer. If the incident angles remain roughly constant for a short time period, we can assume that the antenna weights need not be changed during that period.

thus separating the weights from the other variables. It can be shown that $\Gamma F=L(\mathcal{W}) \cdot M(\Gamma, \mathcal{G}, \mathcal{A}) \cdot R(\mathcal{W})$, [8]. where the sets inside the parenthesis show the dependency of each matrix on each collection of variables.

Now we use these matrices to answer our convergence question: $\|\Gamma F\|_{1}<1$. We can state that

$$
\|L\|_{1} \cdot\|R\|_{1}<\frac{1}{\|M\|_{1}} \Rightarrow\|\Gamma F\|_{1}<1 .
$$

We must, therefore, find a set of vectors, $\left\{w_{i}\right\}_{i=1, \cdots M}$, that comply with the left inequality in (1). If there are any such vectors, then we have convergence. We must, therefore, find a set of vectors that minimize $\|L\|_{1} \cdot\|R\|_{1}$.

Theorem 1: The set of weight vectors, $\left\{\mathbf{w}_{i}\right\}_{i=1 \cdots M}$, that minimizes $\|L\|_{1} \cdot\|R\|_{1}$, while $\mathbf{w}_{i}^{H} \mathbf{a}_{i i}=1$ is

$$
\tilde{w}_{i}(k)= \begin{cases}\frac{1}{a_{i, \max }^{*}} & , k=\arg \max _{j=1, \ldots D}\left|\mathbf{a}_{i i}(j)\right| \\ 0 & , \text { otherwise }\end{cases}
$$

where $a_{i, \max }=\mathbf{a}_{i i}(k)$, and $D$ is the number of antenna elements at each base station.

Proof: [8]

\section{B. SINR Fine-tuning}

Once all users are functioning, we may devote our computing resources to improving and fine-tuning the SINR of multimedia users. Since we work off-line, we can use more computationally expensive methods.

We wish to bring as many $\left\{\gamma_{i}\right\}_{i \in I}$ as we can towards their target levels, without driving $\rho(\Gamma F)$ to $I . I$ is the set of indices of the multimedia users. The tool that we use to choose which SINR levels to adjust is $\frac{\partial \rho(\Gamma F)}{\partial \gamma_{i}}$. Let's first assume that these derivatives exist, and we have sorted them from the smallest to the largest. We single out $\gamma_{i_{*}}$ for $i_{\star}=\arg \min _{i \in I} \frac{\partial \rho}{\partial \gamma_{i}}$.

Once the $i_{\star}$ is selected, we increase $\gamma_{i *}$ and test for convergence. Now, we assume that in the small span of time in which this takes place, the positions of the mobiles do not change significantly, see Fig. 2. This is important because we assume that the antenna weights need not be recalculated. We only wish to find a new power vector that is more favorable to user $i_{\star}$, while not chastising the other users. To do this, we can use existing techniques [6] that quickly give us a new power vector, and here, we use the more exact test: $\rho\left(\Gamma_{n e w} F\right)<1$. If the test still passes, we can find a new power vector, and implement it. If the test fails, we reduce the SINR for a different user: $i^{\star}=\arg \max _{i \in I} \frac{\partial \rho}{\partial \gamma_{i}}$. 


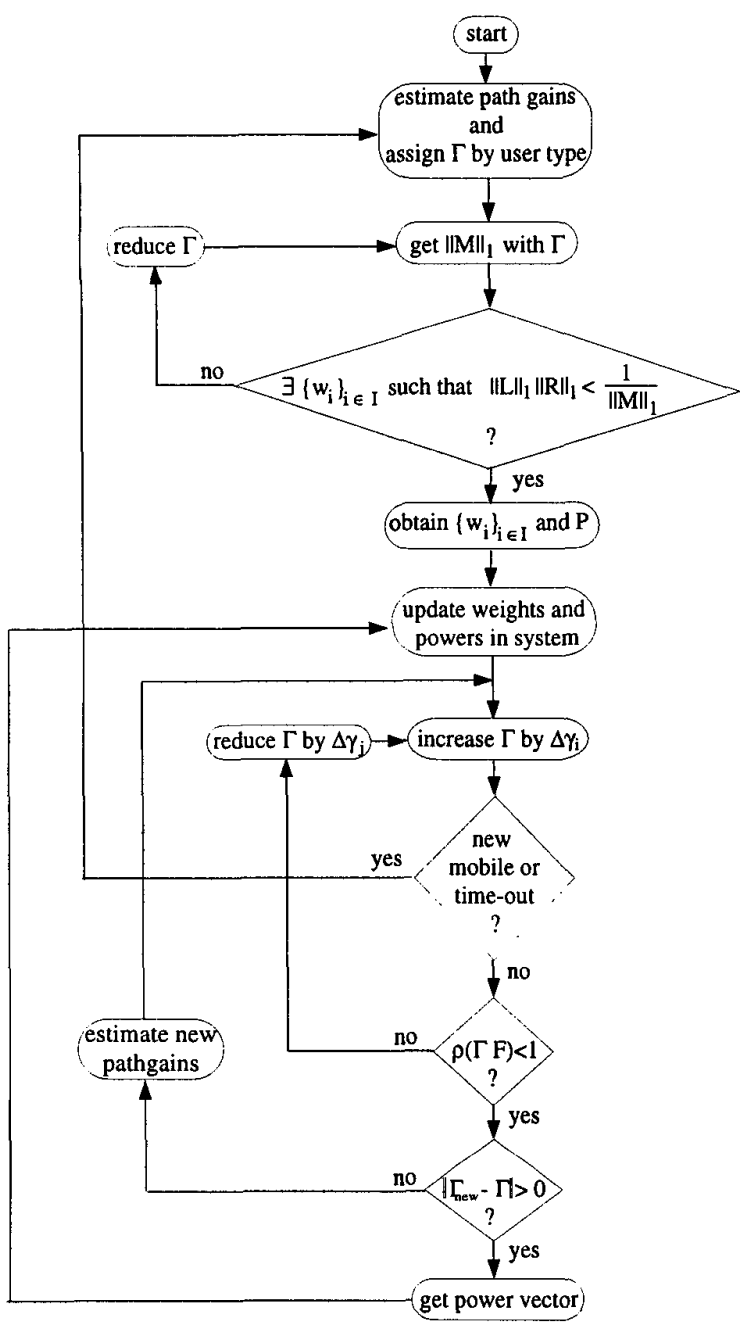

Fig. 3. Flowchart for the algorithm.

\section{Choosing which SINR to Adjust}

In this section, we present the way to obtain $\frac{\partial \rho(\Gamma F)}{\partial \gamma_{i}}$. The question of the existence of the derivative of the spectral radius of such a matrix is addressed in [12], [10].

Theorem 2: For the matrix $\Gamma F$, and the SINR level $\gamma_{i}$ as described in Section II,

$$
\frac{\partial \rho(\Gamma F)}{\partial \gamma_{i}}=\frac{\mathbf{y}^{H} F_{i} \mathbf{x}}{\mathbf{y}^{H} \mathbf{x}},
$$

where $\mathbf{y}$ and $\mathbf{x}$ are the left and right eigenvectors of $\rho$, respectively, and $F_{i}$ is a matrix with the $i^{t h}$ row equal to that of $F$ and zero elsewhere. Proof: $[8] \circ$

It should be noted that the derivative is a measure of infinitesimal change, therefore, we must remind ourselves to choose small perturbations of the $\gamma_{i}$, so that these results may be of use to us. Another reason to keep the perturbations small is that the spectral radius may change from one eigenvalue index to another.

\section{INTEGRATED SINR CONTROL SCHEME}

In this section, we put together the tools described in the previous sections and we formulate a working algorithm. Consider a wireless network whose users are identified according to their multimedia service types. The system will first assign the target SINR levels according to their service types. It will then use the fast and rough test described in Theorem 1 to check if the SINR levels allow convergence. If convergence is allowed, the system will proceed to calculate the mobile powers and the antenna weights, and then implement them. If, however, the test results in failure, the method described in Section III C is used to reduce the SINR level for some user. Once the power vector and antenna weights have been successfully assigned, and the system is using these factors on-line, the processor begins the fine-tuning part of the algorithm.

The fine-tuning steps start by increasing the SINR levels of different high data rate users and testing whether the new SINR levels allow for convergence. In this part, the high rate users that are selected are done so with the criteria shown in Section III C. Here a timer is initiated and the fine-tuning iteration will be confined to work within the constraints of the timer and also it will be interrupted in the case that a new user initiates the service protocol in that channel of that base station. During this part, the system attempts to adjust the power vector, and we assume that the time span is short enough that the antenna weights need not be adjusted. The details of the algorithm can be observed in Fig. 3.

It should be noted that steps should be taken if there is no convergence even after maximum SINR reduction. The maximum SINR reduction is achieved when all users are assigned the minimum SINR level allowable for the proper delivery of each multimedia service type. In such a case, the system should have some user handoff to another frequency, preferably the user that would be handed off would be the one whose index, $j$ has $j=\arg \max _{i \in I} \frac{\partial \rho}{\partial \gamma_{i}}$. In this case, we can consider all the user indexes, and not only the ones in the multimedia subset, $I$. This is because the presence of a voice quality user may be the factor that is most affecting the system at that moment. The call need not be dropped, but only handed off to another frequency, perhaps within the same base station. For simplicity, the algorithm presented in Fig. 3 does not include the steps for forcing a user to hand off.

\section{SiMULATION RESULTS}

We assumed a wireless network with $M=40$ cochannel bases using FDMA, and a $(2,1)$ reuse pattern. Each base station has four antenna elements. We assumed only two user types, voice quality and multimedia quality. The algorithm can be readily extended to several quality types. We assumed that voice users would require $\gamma_{v}$, and they would get that quality, without adjustment. And we assumed that the other users would get $\gamma_{m}$ SINR, which was considerably higher than $\gamma_{v}$ (of the order of $16 d B$ higher). We assume that, on average, $80 \%$ of those $M$ links would be on at any given time, and of the ones that are on, $p=.3$ proportion of them desire a multimedia quality channel. Call lengths are exponentially distributed with an average length of six minutes. The time-out for exiting the fine-tuning stage of the algorithm was set to be 16 seconds times the number of active users.

In all simulations, the voice type users have a fixed SINR level, and the multimedia type users would initially be assigned their target SINR levels. Multimedia type users would have their level reduce from their target level only for the purposes of allowing the calculation of the power vector and antenna weights. No user was allowed to have a SINR level bellow $\gamma_{v}$.

The simulation presented in Figure 4 runs along a time axis. The values that are plotted are: the target SINR for the multimedia users, $\gamma_{m}=32 d B$ which is labeled "desired", the average SINR level for multimedia users if the proposed algorithm described in Section IV is implemented, which is labeled "proposed algorithm with variable SINR". The average SINR over time for the multimedia type users in that data line was $31.5 \mathrm{~dB}$. The line labeled "existing algorithm with single SINR for all users" displays the SINR level for all multimedia type users if the system chooses to have all multimedia SINR levels 


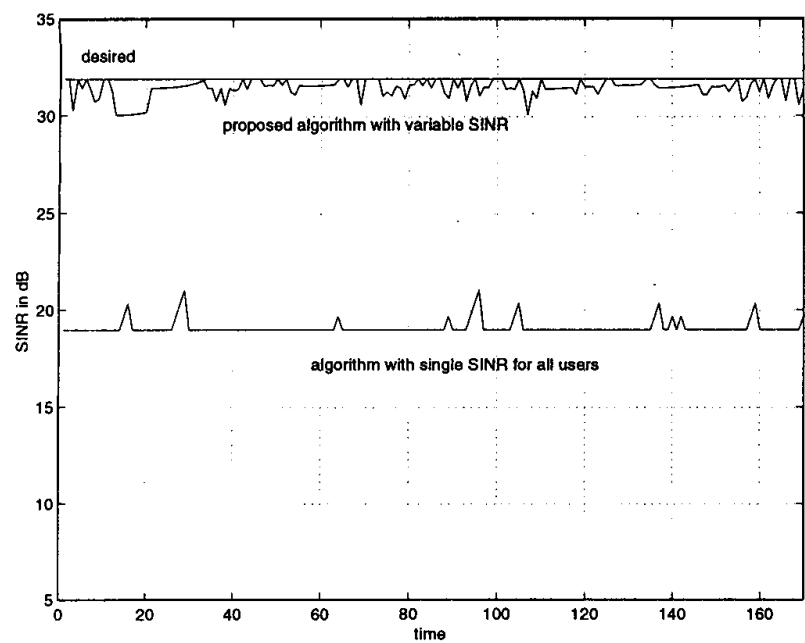

Fig. 4. The average SINR using a constant SINR for all users and raising and lowering them at the same time was $12.3 d B$ lower than with our algorithm.

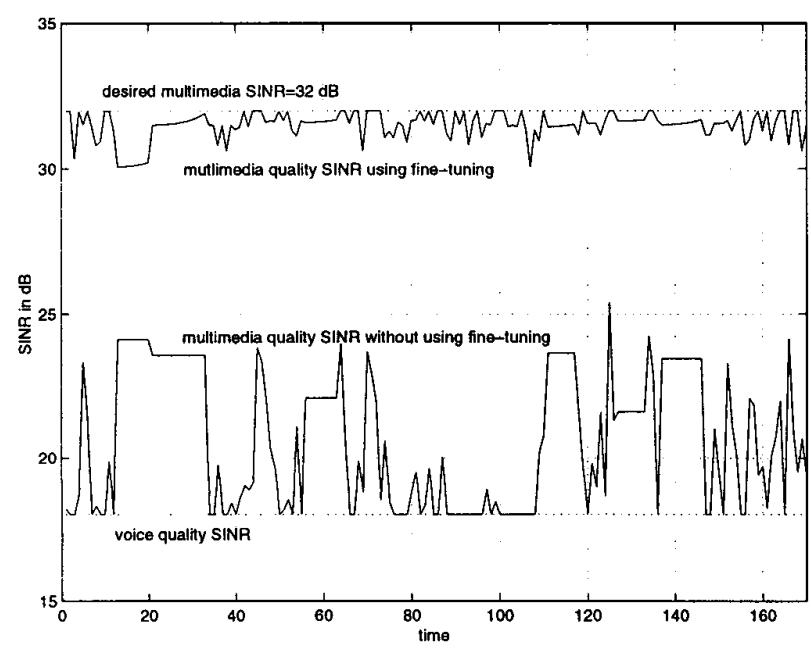

Fig. 5. $p=.3 ; \gamma_{v}=18 d B ; \gamma_{m}=32 d B$. The average difference between the method without fine-tuning and that with fine-tuning was $11.6 \mathrm{~dB}$.

equal. In such a case, the system would initiate the calculations by setting the SINR levels for all users at $\gamma_{m}=32 d B$, and then it would test for convergence. If the convergence test failed, the system would reduce the SINR levels for all multimedia users by a small, equal amount, and then test again. This last line had an average over time of 19.2dB, which is over $12 d B$ lower than the average for our method.

The simulation presented in Fig. 5 displays the importance of the fine-tuning part of the algorithm. As can be seen, the algorithm without the benefit of fine-tuning varies mostly between the voice level SINR of $18 d B$, and about $24 d B$. The fine-tuning part keeps the average SINR for multimedia type users close to the target level of $\gamma_{m}=32 d B$.

Fig. 6 shows the improvement provided by our suggested fast activation method. The values plotted are the times that it takes to calculate the mobile powers and antenna weights when new users are being added to the system. With the existing trial and error method, the computation time was almost two orders of magnitude longer than with

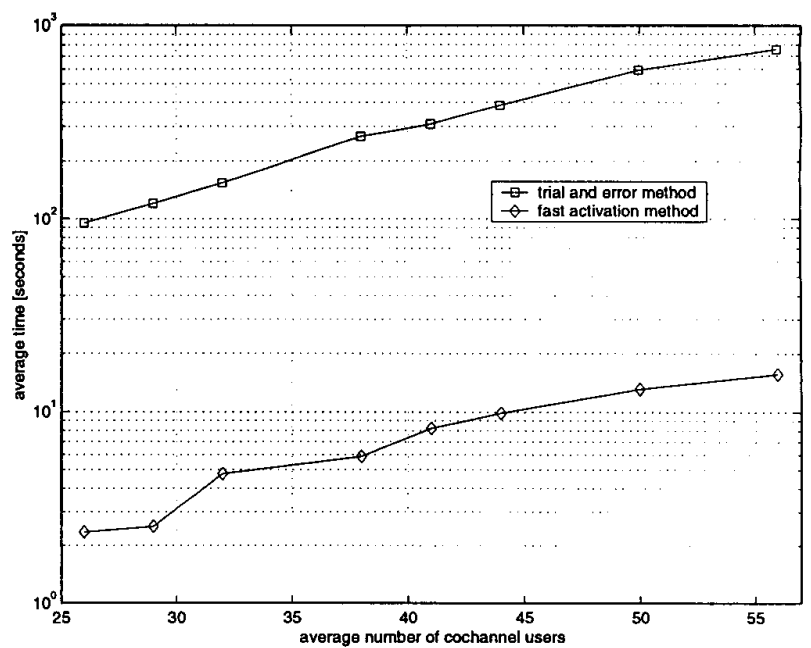

Fig. 6. Average time to activate new users as a function of the average number of active users.

our method. In the existing trial and error scheme, the desired $\gamma$ s are assigned to the new users. The system then engages in an iterative algorithm to get the powers and antenna weights. If the algorithm did not converge in $4 * M$ iterations, it would reduce the SINR for a randomly chosen multimedia user and try again. Here we refer to the average number of cochannel users because approximately $80 \%$ of the total users are on at any given time, but the exact number changes with time as units activate and deactivate.

\section{CONCLUSIONS}

We can gain a significant increase in the SINR for mobile clients that use multimedia services by estimating the spectral properties of the system matrix and using those properties to adjust the power for the mobile units. Our simulations show over $10 \mathrm{~dB}$ of improvement of the SINR by using the proposed SINR fine-tuning scheme.

We've also managed to reduce the latency time for new users significantly by using a coarser and faster method for finding feasible SINR. Our simulations have shown an improvement over a factor of 15 in the time required to find a feasible solution.

\section{REFERENCES}

[1] J. Miller and S. Miller. Smart Antenna Adaptive Performance in the Presence of Imperfect Power Control, Multipath and Shadow Fading. IEEE Global Telecommunications Conference. pages pp. 384-388, November 1997.

[2] J. Winters; C. Martin; and N. Sollenberger. "Forward Link Smant Antennas and Power Control for IS-136", 48th IEEE Vehicular Tec/inology Conference, pages pp. 601-605, May 1998.

[3] Y. Liang; F. Chin; and K.J.R. Liu. "Downlink Beamforming for DS-CDMA Mobile Radio with Multimedia Services". Proc, of IEEE VTS SOth Vehicular Teclinology Conference, pages pp. 17-21, 1999.

[4] A. Grandhi; R. Yates; D. Goodman. "Resource Allocation for Cellular Radio Systems". IEEE Transactions on Veficular Technology, 46(3):pp. 581-587, August 1997.

[5] S. Rappapon; C. Purzynski. "Prioritized Resource Assignment for Mobile Cellular Communication Systems with Mixed Services and Platform Types". IEEE Transacrions on Vehicular Tecinology. 45(3):pp. 443-458, August 1996.

[6] F. Rashid-Farrokhi; K.J.R. Liu; and L. Tassiulas. "Transmit Beamforming and Power Cuntrol ion Cellular Wireless Systems". IEEE Joumal on Selecred Areas in Communications, 16(8):pp. 14371449. October 1998.

[7] R. Monzingo and T. Miller. Introduction to Adaptive Arrays. Wiley - Interscience, 1980 .

[8] A. Mercado; K.J.R. Liu. Adaptive QoS for Multimedia Services for Wireless Networks Using Power Control and Sman Antennas. submitred for publication, IEEE Transactions on Vehicular Techlnology. November 1999.

[9] F. Gantmacher. The Theory of Murrices, volume 2. Chetsea Publishing Company, 1959

[10] R. Bellman. Introduction to Matrix Analysis, Second Edition. SLAM, 1997.

(II) A. Mercado; K.J.R. Liu. NP-Completeness of the Stable Rank One Penturbed Matrix Problem in Discrete-Time. submitted for publication. Systems and Control Lenters, November 1999.

[12] G. Stewart and J. Sun. Matrix Perturbation Theory. Academic Press, 1990. 